\title{
GRAPHS WITH MAXIMAL NUMBER OF ADJACENT PAIRS OF EDGES
}

\author{
By \\ R. AHLSWEDE ${ }^{1}$ (Bielefeld) and G. O. H. KATONA ${ }^{2}$ (Budapest)
}

\section{Introduction and statement of the results}

Let $G_{n}^{N}$ denote an undirected graph (without loops and multiple edges) with $n$ vertices and $N$ edges. $P\left(G_{n}^{N}\right)$ shall count the number of pairs of different edges which have a common vertex and finally $f(n, N)$ is defined by

$$
f(n, N)=\max p\left(G_{n}^{N}\right),
$$

where the maximum is taken over all possible graphs $G_{n}^{N}$.

In information theory the problem came up to determine $f(n, N)$ for certain hypergraphs. We give here a solution for graphs and for bipartite graphs. As Vera T. Sós kindly informed us, this problem has been solved by MosHE KATZ [1] for “nice" $N$ 's.

In order to state our results we need the concepts of a quasi-complete graph and of a quasi-star. Suppose the vertices of the graph are denoted by $1,2, \ldots, n$. We define the quasi-complete graph $C_{n}^{N}$ with $N$ edges in the following way: $i$ and $j$ are connected for $i, j \leqq a(i \neq j)$ and $a+1$ is connected with $1,2, \ldots, b$, where $a$ and $b$ are determined by the unique representation

$$
N=\left(\begin{array}{l}
a \\
2
\end{array}\right)+b, \quad 0 \leqq b<a .
$$

A quasi-star $S_{n}^{N}$ with $N$ edges is defined as follows: use the unique representation

$$
\left(\begin{array}{l}
n \\
2
\end{array}\right)-N=\left(\begin{array}{l}
c \\
2
\end{array}\right)+d, \quad 0 \leqq d<c
$$

and connect the first $n-c-1$ vertices with every other, connect the vertex $n-c$ with the first $n-d$ vertices.

It is easy to see that $S_{n}^{\left(\begin{array}{c}n \\ 2\end{array}\right)-N}$ is the complement graph of $C_{n}^{N}$ if we change the order of the vertices. We use the abbreviations

$$
C(n, N)=p\left(\begin{array}{l}
N \\
n
\end{array}\right), \quad S(n, N)=p\left(S_{n}^{N}\right) .
$$

Let $G_{l, m}^{N}$ denote an aritrary bipartite graph with $N$ edges and $l+m$ vertices, where $l$ vertices are coloured red and $m$ pink.

${ }^{1}$ Research of this author was supported by the Deutsche Forschungsgemeinschaft.

2 This work was done while the author visited the Institute of Mathematical Statistics, University of Göttingen. 
THEOREM 1. Suppose $1 \leqq m, N=q m+r, 0 \leqq r<m$. Then $\max p\left(G_{i, m}^{N}\right)$ is assumed for the graph in which $q$ red vertices are all connected with all the pink ones and one more red vertex is connected with $r$ pink ones.

THEOREM 2. $f(n, N)=\max [C(n, N), S(n, N)]$.

THEOREM 3.

$$
f(n, N)=\left\{\begin{array}{lll}
S(n, N) & \text { if } & 0 \leqq N<\frac{1}{2}\left(\begin{array}{l}
n \\
2
\end{array}\right)-\frac{n}{2} \\
C(n, N) & \text { if } & \frac{1}{2}\left(\begin{array}{l}
n \\
2
\end{array}\right)+\frac{n}{2}<N \leqq\left(\begin{array}{l}
n \\
2
\end{array}\right) .
\end{array}\right.
$$

Moreover, there are infinitely many $n$ 's for which $f(n, N)=S(n, N)$ for all $N<\frac{1}{2}\left(\begin{array}{l}n \\ 2\end{array}\right)$ and $f(n, N)=C(n, N)$ for all $N>\frac{1}{2}\left(\begin{array}{l}n \\ 2\end{array}\right)$. On the other hand there are infinitely many $n$ 's for which this is not true. (For further details see Lemma 8.)

We give two different proofs of Theorem 2. The first is more elegant and is based on Theorem 1 .

The second proof is more elaborate, however, it uses techniques which we also use in the proof of Theorem 3, and it is worthwhile knowing that both Theorems can be proved by the same approach. The first proof might be more suited for generalizations of Theorem 2 .

\section{Proof of Theorem 1}

The present proof and also the first proof of Theorem 2 are formulated in terms of vertex-vertex incidence matrices.

For the bipartite graph $G_{l, m}^{N}$ with $l$ red and $m$ pink vertices and with $N$ edges the matrix $J\left(G_{i, m}^{N}\right)$ is defined by

where

$$
J\left(G_{i, m}^{N}\right)=\left(a_{i j}\right)_{j=1, \ldots, m}^{i=1, \ldots, l}
$$

$$
a_{i j}= \begin{cases}1 & \text { if the } i \text {-th red and } j \text {-th pink vertices are connected in } G_{l, m}^{N} \\ 0 & \text { otherwise. }\end{cases}
$$

If $J_{l, m}^{N}$ is an $l \times m 0-1$-matrix with $N$ 1's $q\left(J_{l, m}^{N}\right)$ denotes

$$
\sum_{i=1}^{l} r_{i}^{2}+\sum_{i=1}^{m} s_{i}^{2}
$$

where $r_{1}, \ldots, r_{l}$ and, $s_{1}, \ldots, s_{m}$ are the numbers of 1's in the rows and columns, respectively. Since

$$
q\left(J\left(G_{i, m}^{N}\right)\right)=2 p\left(G_{i, m}^{N}\right)+2 N,
$$

for our purposes it suffices to maximize the quadratic form $q\left(J_{i, m}^{N}\right)$. We need

LEMMA 1. $\max q\left(J_{i, m}^{N}\right)$ is assumed for a matrix with the property: if $a_{i j}=1$ and $i^{\prime} \leqq i, j^{\prime} \leqq j$, then $a_{i^{\prime} j^{\prime}}=1$. 
Proof. Suppose $J_{l, m}^{N}$ maximizes and its rows and columns are ordered such that $r_{1} \geqq r_{2} \geqq \ldots \geqq r_{l}$ and $s_{1} \geqq s_{2} \geqq \ldots \geqq s_{m}$. Suppose that there are entries $a_{i j}=1$, $a_{i j^{\prime}}=0$ for $j^{\prime}<j$ (or $a_{i^{\prime} j}=0$ for $i^{\prime}<i$ ). By exchanging this 1 and $0, q\left(J_{l, m}^{N}\right)$ changes by

$$
-s_{j^{\prime}}^{2}+\left(s_{j}-1\right)^{2}+\left(s_{j^{\prime}}+1\right)^{2}-s_{j^{\prime}}^{2}=2+2\left(s_{j^{\prime}}-s_{j}\right)>0,
$$

a contradiction and the lemma is proved.

Proof of Theorem 1. We prove it by induction on $n=l+m$. If $l+m=2$ the statement is trivial. Suppose that $l+m>2$ and also that $J_{l, m}^{N}$ has the properties described in Lemma 1. We can also assume that $r_{1} \geqq s_{1}$, because otherwise we can exchange the role of rows and columns without changing the total number of rows and columns, because $r_{1}<s_{1} \leqq m$, and we can have 1's only in the first $r_{2}$ columns. (We write 0 's in the undefined places.)

Notice that we have now 1's only in the submatrix $J^{*}$ determined by the first $r_{1}$ columns and first $s_{1}$ rows, and by Lemma 1 the first row of this submatrix contains only 1's. Denoting by $J_{s_{1}-1, r_{1}}^{N-r_{1}}$ the $\left(s_{1}-1\right) \times r_{1}$-matrix, which is derived form $J^{*}$ by omitting its first row, we can establish the recursion formula

$$
\begin{gathered}
q\left(J_{l, m}^{N}\right)=\sum_{i=1}^{m} s_{i}^{2}+\sum_{i=1}^{l} r_{i}^{2}=\sum_{i=1}^{r_{1}} s_{i}^{2}+\sum_{i=1}^{s_{1}} r_{i}^{2}= \\
=\left(\sum_{i=1}^{r_{1}}\left(s_{i}-1\right)^{2}+\sum_{i=2}^{c_{1}} r_{i}^{2}\right)+\sum_{i=1}^{r_{1}}\left(2 s_{i}-1\right)+r_{1}^{2}=q\left(J_{s_{1}-1, r_{1}}^{N-r_{1}}\right)+2 N-r_{1}+r_{1}^{2} .
\end{gathered}
$$

This means that if we want to maximize $q\left(J_{i, m}^{N}\right)$ with fixed $r_{1}$ and $c_{1}$ then we have to maximize $q\left(J_{s_{1}-1, r_{1}}^{N-r_{1}}\right)$. Here $s_{1}-1+r_{1}<l+m$ and we can use the induction hypothesis. Since $c_{1}-1<r_{1}, \max q\left(J_{s_{1}-1, r_{1}}^{N-r_{1}}\right)$ is assumed for a matrix in which the first $u-1$ rows are full with 1 's and in the $u$-th row the first $r$ entries are 1 's, where

$$
N-r_{1}=(u-1) r_{1}+v, \quad 0 \leqq v<r_{1} .
$$

All the other entries are 0's.

We have thus proved that it is sufficient to consider the matrices $J_{l, m}^{N}$ which have 1's in the first $r_{1}$ places of the first $u$ rows and in the first $v$ places in the $(u+1)$-th row, where $u \leqq r_{1} \leqq m$ and $N=u r_{1}+v, 0 \leqq v<r_{1}$. Denote these matrices by $J\left(N, r_{1}\right)$. We have only to prove that

$$
q\left(J\left(N, r_{1}+1\right)\right) \geqq q\left(J\left(N, r_{1}\right)\right) .
$$

For this we use the equation

$$
q\left(J\left(N, r_{1}\right)\right)=u_{1}^{2}+v^{2}+v(u+1)^{2}+\left(r_{1}-v\right) u^{2} .
$$

We distinguish two cases:

1. Case $v \geqq u$. Then

and by (2.6)

$$
N=u\left(r_{1}+1\right)+v-u, \quad 0 \leqq v-u<r
$$

$$
q\left(j\left(N, r_{1}+1\right)\right)=u\left(r_{1}+1\right)^{2}+(v-u)^{2}+(v-u)(u+1)^{2}+\left(r_{1}-v+u+1\right) u^{2} .
$$


An elementary calculation yields

$$
q\left(J\left(N, r_{1}+1\right)\right)-q\left(J\left(N, r_{1}\right)\right)=2 r_{1} u-2 v u .
$$

Since $r_{1}>v$ this proves $(2.5)$ in the first case.

2. Case $v<u$. Then $N=(u-1)\left(r_{1}+1\right)+\left(v-u+r_{1}+1\right)$, where $0 \leqq v-u+r_{1}+1<$ $<r_{1}+1$ follows from $u \leqq r_{1}$ and $v<u$. We can again use (2.6) and get

$$
\begin{gathered}
q\left(J\left(N, r_{1}+1\right)\right)=(u-1)\left(r_{1}+1\right)^{2}+\left(v-u+r_{1}+1\right)^{2}+\left(v-u+r_{1}+1\right) u^{2}+ \\
+(u-v)(u-1)^{2} .
\end{gathered}
$$

Again by an elementary calculation

$$
q\left(J\left(N, r_{1}+1\right)\right)-q\left(J\left(N, r_{1}\right)\right)=2 r_{1} v-2 u v,
$$

which is non-negative because $r_{1} \geqq u$. We thus have proved (2.5) in both cases and $q\left(J\left(N, r_{1}\right)\right)$ is maximal if we choose $r_{1}=m$. The Theorem is proved.

\section{First proof of Theorem 2}

Denote the vertex-vertex incidence matrix of $G_{n}^{N}$ by

where

$$
I\left(G_{n}^{N}\right)=\left(a_{i j}\right)_{i=1, \ldots, n}^{j=1, \ldots, n}
$$

$$
a_{i j}= \begin{cases}1 & \text { if } i \text { and } j \text { are connected } \\ 0 & \text { otherwise. }\end{cases}
$$

The $n \times n$-matrix $I\left(G_{n}^{N}\right)$ is symmetric, has $2 N$ 1's and 0 's in the diagonal. Also every $0-1$-matrix $I_{n}^{N}$ is the incidence matrix of a graph $G_{n}^{N}$. Define

$$
Q\left(I_{n}^{N}\right)=\sum_{i=1}^{n} c_{i}^{2}
$$

where $c_{i}$ counts the number of 1's in the $i$-th row or $i$-th column. Clearly,

$$
Q\left(I\left(G_{n}^{N}\right)\right)=2 p\left(I\left(G_{n}^{N}\right)\right)+2 N
$$

and it suffices to maximize the expression in (3.1). Again we need an auxiliary result

Lemma 2. $\max Q\left(I_{n}^{N}\right)$ is assumed for a matrix with the property: if $a_{i j}=1$ and $i^{\prime} \leqq i, j^{\prime} \leqq j, i^{\prime} \neq j^{\prime}$, then $a_{i^{\prime} j^{\prime}}=1$.

Proof. Let the rows and columns of an optimal $I_{n}^{N}$ be numbered by $1,2, \ldots, n$ such that $c_{1} \geqq c_{2} \geqq \ldots \geqq c_{n}$. Now suppose that $a_{i j}=1, i<j$, but $a_{i^{\prime} j}=0$ for $i^{\prime}<i$. By exchanging this 1 and 0 (also symmetrically the corresponding term) we get

$$
c_{i^{\prime}}^{*}=c_{i^{\prime}}+1, \quad c_{i}^{*}=c_{i}-1, \quad c_{t}^{*}=c_{t} \text { for } t \neq i^{\prime}, i,
$$


and

$$
\sum_{t=1}^{n} c_{t}^{* 2}-\sum_{t=1}^{n} c_{t}^{2}=2 c_{i}^{\prime}-2 c_{i}+2>0,
$$

because $c_{i}^{\prime} \geqq c_{i}$. This contradiction proves the lemma.

We begin now the proof of Theorem 2. Suppose $I_{n}^{N}$ has the form described in Lemma 2. Denote the largest index $i$ satisfying $a_{i, i-1}=1$ by $w=w\left(I_{n}^{N}\right)$. Then

$$
\left\{\begin{array}{l}
a_{w+1, w}=a_{w, w+1}=0, \quad a_{i j}=0, \quad \text { for } w+1 \leqq i, j . \\
a_{i j}=1 \text { for } 1 \leqq i, j \leqq w \text { with } i \neq j .
\end{array}\right.
$$

In the class of matrices $I_{n}^{N}$ maximizing $Q\left(I_{n}^{N}\right)$, let the minimal and maximal value of $w\left(I_{n}^{N}\right)$ be denoted by $w_{1}$ and $w_{2}$, respectively. We distinguish two cases:

1. Case $w_{2} \geqq \frac{n}{2}$. Cut the matrix $I_{n}^{N}$, with $w_{2}=w\left(I_{n}^{N}\right)$, after the $w_{2}$-th row and $w_{2}$-th column. It is separated now into four submatrices $A, B, C$ and $D$. Here $A$ is a $w_{2} \times w_{2}$ matrix with 0 's only in the diagonal. $B$ and $C$ are $w_{2} \times\left(n-w_{2}\right)$ and $\left(n-w_{2}\right) \times w_{2}$ matrices (symmetrical images of each other), respectively, $D$ is a 0 -matrix.

Consider the expression

$$
\begin{aligned}
Q\left(I_{n}^{N}\right) & =\sum_{i=j}^{n} c_{i}^{2}=\sum_{i=1}^{w_{2}}\left(c_{i}-\left(w_{2}-1\right)\right)^{2}+2 \sum_{i=1}^{w_{2}} c_{i}\left(w_{2}-1\right)-\sum_{i=1}^{w_{2}}\left(w_{2}-1\right)^{2}+\sum_{i=w_{2}+1}^{n} c_{i}^{2}= \\
& =\sum_{i=1}^{w_{2}}\left(c_{i}-\left(w_{2}-1\right)\right)^{2}+\sum_{i=w_{2}+1}^{n} c_{i}^{2}+2 N\left(w_{2}-1\right)-2 w_{2}\left(w_{2}-1\right)^{2} .
\end{aligned}
$$

$B$ and $C$ are incidence matrices of bipartite graphs and

$$
q(B)=q(C)=\sum_{i=1}^{w_{2}}\left(c_{i}-\left(w_{2}-1\right)\right)^{2}+\sum_{i=w_{2}+1}^{n} c_{i}^{2} .
$$

To maximize $Q\left(I_{n}^{N}\right)$ for fixed $w_{2}$ means therefore simply to maximize $q(B)$. Since $w_{2} \geqq n-w_{2}$ it follows from Theorem 1 that the first column of $B$ contains as many 1 's as the number of 1 's in $B$ permits. This leads to a contradiction if the first column is full, because $a_{w_{2}, w_{2}+1}=1$ contradicts the definition of $w_{2}$. It follows that $B$ contains 1's only in the first column and that this number is smaller than $w_{2}$.

$C$ is symmetrical to $B$. The matrix $I_{n}^{N}$, which consists of $A, B, C$ and $D$ is the incidence matrix of a quasi-complete graph.

2. Case $w_{2}<\frac{n}{2}$. In this case also $w_{1}<\frac{n}{2}$. Cut now the matrix $I_{n}^{N}$ with $w_{1}=w\left(I_{n}^{N}\right)$ after the first $w_{1}-1$ rows and $w_{1}-1$ columns. We obtain four matrices: $A, B, C$ and $D$. $A$ is a $\left(w_{1}-1\right) \times\left(w_{1}-1\right)$ matrix with 0 's only in the diagonal. $B$ is a $\left(w_{1}-1\right) \times\left(n-w_{1}+1\right)$ matrix, $C$ is the symmetrical image of $B$. $D$ is a 0 -matrix since $a_{w_{1}, w_{1}+1}=0$. We can use (3.4) with $w_{1}-1$ instead of $w_{2}$. Then $Q\left(I_{n}^{N}\right)$ can be maximized by maximizing $q(B)$. Since $w_{1}-1<n-w_{1}+1$ by Theorem $1, q(B)$ is maximal if we choose $B$ full with 1's in the first rows. However, by definition of $w_{1}, a_{w_{1}-1, w_{1}}=1$, that is, the first element of the last row of $B$ is 1 . It follows that 
the first $w_{1}-2$ rows of $B$ are full with 1's, because otherwise we could find a $B$ (and $I_{n}^{N}$ ) which maximizes $Q\left(I_{n}^{N}\right)$ and has $w\left(I_{n}^{N}\right)<w_{1}$.

Thus we have found an optimal matrix which has 1's in the first $w_{1}-2$ rows and columns everywhere except the diagonal, furthermore it has 1's at the beginning of the $\left(w_{1}-1\right)$-th row and column. The other entries are 0 . This is the incidence matrix of a quasi-star graph. Case 1 and 2 yield

$$
f(n, N)=\max (C(n, N), S(n, N)) .
$$

Notice that the proof gives also an interesting property of $S(n, N)$ :

$$
S(n+1, N) \geqq S\{n, N) \quad \text { if } \quad n \geqq 4 \text { and } N \leqq \frac{1}{2}\left(\begin{array}{l}
n \\
2
\end{array}\right) .
$$

To see this it suffices to check that $w\left(S_{n}^{N}\right)<\frac{n+1}{2}$. Suppose the contrary, then

$$
\begin{aligned}
& 2 N \geqq w(w-1)+2(n+1-w)(w-2)=-w^{2}+(2 n+5) w-4(n+1) \geqq \\
& \geqq-\left(\frac{n+1}{2}\right)^{2}+(2 n+5) \frac{n+1}{2}-4(n+1)=\frac{(n+1)(3 n-7)}{4}>\frac{n(n-1)}{2} .
\end{aligned}
$$

This contradiction for $n \geqq 4$ establishes (3.6).

\section{Comparison of $C(n, N)$ and $S(n, N)$, the proof of Theorem 3}

At the first moment one might think that it should be easy to compare $C(n, N)$ and $S(n, N)$ for given $n$ and $N$. However, the functions are given only in an implicit way by number-theoretical-combinatorial expressions. Also around $N=\frac{1}{2}\left(\begin{array}{l}n \\ 2\end{array}\right)$ they are very close to each other. Of course it is quite easy to make the comparison if $N \ll \frac{1}{2}\left(\begin{array}{l}n \\ 2\end{array}\right)$ or $N \gg \frac{1}{2}\left(\begin{array}{l}n \\ 2\end{array}\right)$, but we would like to consider values of $N$ around $\frac{1}{2}\left(\begin{array}{l}n \\ 2\end{array}\right)$ as well. We shall need several lemmas, which we now state and prove.

Lemma 3.

a) $S(n, N)=C\left(n,\left(\begin{array}{l}n \\ 2\end{array}\right)-N\right)-n\left(\begin{array}{c}n-1 \\ 2\end{array}\right)-4 N+2 n N$

b) $S(n, N)-C(n, N)=C\left(n,\left(\begin{array}{l}n \\ 2\end{array}\right)-N\right)-S\left(n,\left(\begin{array}{l}n \\ 2\end{array}\right)-N\right)$.

Proof. Since the quasi-star with $N$ edges and the quasi-complete graph with $\left(\begin{array}{l}n \\ 2\end{array}\right)-N$ edges are complementary to each other (if we change the order of the vertices), it suffices to prove the statements for any pair of complementary graphs. If we denote by $c_{1}, \ldots, c_{n}$ the valencies of the first graph, then $\sum_{i=1}^{n} c_{i}=2 N, \sum_{i=1}^{n}\left(\begin{array}{l}c_{i} \\ 2\end{array}\right)$ 
is the number of adjacencies and $\sum_{i=1}^{n}\left(\begin{array}{c}n-c_{i}-1 \\ 2\end{array}\right)$ is the corresponding number for the complement graph. Now

$$
\begin{gathered}
\sum_{i=1}^{n}\left(\begin{array}{c}
n-c_{i}-1 \\
2
\end{array}\right)=\frac{1}{2} \sum_{i=1}^{n}\left(n-c_{i}-1\right)\left(n-c_{i}-2\right)= \\
=\frac{1}{2} \sum_{i=1}^{n} n^{2}-\sum_{i=1}^{n} n c_{i}-\frac{3}{2} \sum_{i=1}^{n} n+2 \sum_{i=1}^{n} c_{i}+\sum_{i=1}^{n} 1+\frac{1}{2} \sum_{i=1}^{n}\left(c_{i}^{2}-c_{i}\right)= \\
=\frac{n^{3}}{2}-2 N n-\frac{3}{2} n^{2}+4 N+n+\sum_{i=1}^{n}\left(\begin{array}{c}
c_{i} \\
2
\end{array}\right)=\sum_{i=1}^{n}\left(\begin{array}{c}
c_{i} \\
2
\end{array}\right)+n\left(\begin{array}{c}
n-1 \\
2
\end{array}\right)+4 N-2 n N .
\end{gathered}
$$

b) follows easily from a).

After we know now that one of the functions can be expressed in terms of the other one, we express now $C(n, N)$ as partial sum of an infinite sequence. Define $\beta_{i j}$ by

$$
\beta_{i j}=i+j, \quad 0 \leqq j \leqq i .
$$

The $k$-th element of the sequence $\beta_{00}, \beta_{10}, \beta_{20}, \beta_{21}, \beta_{22}, \ldots$ is denoted by $\alpha_{k}$.

Lemma 4. $C(n, N)=\sum_{k=1}^{N} \alpha_{k}$.

Proof. We proceed by induction on $N$. The statement clearly holds for $N=1$. Use the expansion

$$
N=\left(\begin{array}{l}
a \\
2
\end{array}\right)+b, \quad 0 \leqq b<a .
$$

It is easy to see that $\alpha_{N}=\beta_{a-1, b-1}$. Recall that the quasi-complete graph with $N$ edges is composed out of a complete graph of $a$ vertices and an additional $(a+1)$-st vertex, which is connected with the first $b$ vertices.

Suppose that $b+1<a$, then the quasi-complete graph with $N+1=\left(\begin{array}{l}a \\ 2\end{array}\right)+b+1$ $(0 \leqq b+1<a)$ has one more edge from the $(a+1)$-st vertex. The number of new adjacencies is $a-1+b$. This is, indeed, equal to the new term $\alpha_{N+1}=\beta_{a-1, b}$. If $b+1=a$, then the quasi-complete graph with $N+1=\left(\begin{array}{c}a+1 \\ 2\end{array}\right)$ edges is simply a complete graph with $a+1$ vertices. The number of new adjacencies is $2 a-2=$ $=\beta_{a-1, a-1}$ which again equals $\alpha_{N+1}$. The proof is complete.

LEMMA 5. a) $C(n, N) \leqq S(n, N)$ is equivalent to

$$
\sum_{k=1}^{N}\left(\alpha_{k}+\alpha_{\left(\begin{array}{c}
n \\
2
\end{array}\right)-k+1}\right) \leqq N(2 n-4) .
$$
b) If $C(n, N) \leqq S(n, N)$ for some $n(\geqq 4), N\left(\leqq \frac{1}{2}\left(\begin{array}{l}n \\ 2\end{array}\right)\right)$ then it is also true
for $n+1, N$. 
Proof. By Lemma 3a), $C(n, N) \leqq S(n, N)$ is equivalent to

$$
C(n, N)+n\left(\begin{array}{c}
n-1 \\
2
\end{array}\right)-C\left(n,\left(\begin{array}{l}
n \\
2
\end{array}\right)-N\right) \leqq N(2 n-4) .
$$

Since

we get from Lemma 4

$$
\sum_{i=1}^{n-2} \sum_{j=0}^{i} \beta_{i j}=n\left(\begin{array}{c}
n-1 \\
2
\end{array}\right)
$$

$$
\left(\begin{array}{c}
n-1 \\
2
\end{array}\right)-C\left(n,\left(\begin{array}{l}
n \\
2
\end{array}\right)-N\right)=\sum_{i=1}^{n-2} \sum_{j=0}^{i} \beta_{i j}-\sum_{k=1}^{\left(\begin{array}{c}
n \\
2
\end{array}\right)-N} \alpha_{k}=\sum_{k=1}^{N} \alpha_{\left(\begin{array}{l}
n \\
2
\end{array}\right)-k+1}
$$

and that (4.1) is equivalent to

$$
\sum_{k=1}^{N}\left(\alpha_{k}+\alpha_{\left(\begin{array}{l}
n \\
2
\end{array}\right)-k+1}\right) \leqq N(2 n-4) .
$$

Obviously b) follows from (3.6) and the fact that $C(n+1, N)=C(n, N)$ for $N \leqq\left(\begin{array}{l}n \\ 2\end{array}\right)$.

We give now a proof which uses only results of the present section. It is far more complicated than the above argument, but it also shows how the new techniques work, which we need later anyhow.

It is clear from a) that it suffices to prove the inequality

or equivalently that

$$
\sum_{k=1}^{N}\left(\alpha_{k}+\alpha_{\left(\begin{array}{c}
n+1 \\
2
\end{array}\right)-k+1}\right) \leqq N(2 n-2)
$$

$$
\sum_{k=1}^{N}\left(\alpha_{\left(\begin{array}{c}
n+1 \\
2
\end{array}\right)-k+1}-\alpha_{\left(\begin{array}{c}
n \\
2
\end{array}\right)-k+1}\right) \leqq 2 N .
$$

We prove it first for some special $N$ 's. Suppose

$$
N=\left(\begin{array}{l}
n \\
2
\end{array}\right)-\left(\begin{array}{l}
l \\
2
\end{array}\right) \quad\left(\left\{\frac{n+1}{2}\right\} \leqq l \leqq n\right)
$$

( $\{x\}$ is the smallest integer $\geqq x$ ). Then

and

$$
\sum_{k=1}^{N} \alpha_{\left(\begin{array}{l}
n \\
2
\end{array}\right)-k+1}=\sum_{i=l-1}^{n-2} \sum_{j=0}^{i} \beta_{i j}=\sum_{i=0}^{n-2} \sum_{j=0}^{i} \beta_{i j}-\sum_{i=0}^{l-2} \sum_{j=0}^{i} \beta_{i j}=n\left(\begin{array}{c}
n-1 \\
2
\end{array}\right)-l\left(\begin{array}{c}
l-1 \\
2
\end{array}\right),
$$

$$
\begin{gathered}
\sum_{k=1}^{N} \alpha_{\left(\begin{array}{c}
n+1 \\
2
\end{array}\right)-k+1}=\sum_{i=l+1}^{n-1} \sum_{j=0}^{i} \beta_{i j}+\sum_{j=n-l}^{l} \beta_{l, j}=\sum_{i=0}^{n-1} \sum_{j=0}^{i} \beta_{i j}-\sum_{i=0}^{l} \sum_{j=0}^{i} \beta_{i j}+\sum_{j=n-1}^{l} \beta_{l, j}= \\
=(n+1)\left(\begin{array}{c}
n \\
2
\end{array}\right)-(l+2)\left(\begin{array}{c}
l+1 \\
2
\end{array}\right)+(2 l-n+1) l+(2 l-n+1) \frac{n}{2} .
\end{gathered}
$$


Therefore the left hand side of (4.5) is

which equals

$$
(n+1)\left(\begin{array}{l}
n \\
2
\end{array}\right)-(l+2)\left(\begin{array}{c}
l+1 \\
2
\end{array}\right)+(2 l-n+1)\left(l+\frac{n}{2}\right)-n\left(\begin{array}{c}
n-1 \\
2
\end{array}\right)+l\left(\begin{array}{c}
l-1 \\
2
\end{array}\right),
$$

We proved that in the case

$$
2\left(\left(\begin{array}{l}
n \\
2
\end{array}\right)-\left(\begin{array}{l}
l \\
2
\end{array}\right)\right)=2 N
$$

$$
N=\left(\begin{array}{l}
n \\
2
\end{array}\right)-\left(\begin{array}{l}
l \\
2
\end{array}\right) \quad\left(\left\{\frac{n+1}{2}\right\} \leqq l \leqq n\right)
$$

exact equality holds in (4.5).

We shall now verify that the difference of the sides (right-left) of (4.5) is an increasing function of $N$ in the interval

and it is decreasing in

$$
\left(\begin{array}{l}
n \\
2
\end{array}\right)-\left(\begin{array}{l}
l \\
2
\end{array}\right) \leqq N \leqq\left(\begin{array}{c}
n+1 \\
2
\end{array}\right)-\left(\begin{array}{c}
l+1 \\
2
\end{array}\right)
$$

$$
\left(\begin{array}{c}
n+1 \\
2
\end{array}\right)-\left(\begin{array}{c}
l+1 \\
2
\end{array}\right) \leqq\left(\begin{array}{c}
n \\
2
\end{array}\right)-\left(\begin{array}{c}
l-1 \\
2
\end{array}\right) \text {. }
$$

In the last interval (4.6) the last terms of the left hand side of (4.5) are

and

$$
\alpha_{\left(\begin{array}{c}
n+1 \\
2
\end{array}\right)-N+1}=\beta_{l,\left(\begin{array}{c}
n+1 \\
2
\end{array}\right)-\left(\begin{array}{c}
++1 \\
2
\end{array}\right)-N}
$$

$$
\alpha_{\left(\begin{array}{c}
n \\
2
\end{array}\right)-N+1}=\beta_{l-2, l-1-N+\left(\begin{array}{l}
n \\
2
\end{array}\right)-\left(\begin{array}{l}
l \\
2
\end{array}\right)}\left(\beta_{l-1,0} \quad \text { if } \quad N=\left(\begin{array}{l}
n \\
2
\end{array}\right)-\left(\begin{array}{l}
l \\
2
\end{array}\right)\right) .
$$

Thus for $N+1$, the new terms are

Their difference is

$$
\beta_{l,\left(\begin{array}{c}
n+1 \\
2
\end{array}\right)-\left(\begin{array}{c}
l+1 \\
2
\end{array}\right)-N-1} \text { and } \beta_{l-2, l-2-N+\left(\begin{array}{c}
n \\
2
\end{array}\right)-\left(\begin{array}{l}
l \\
2
\end{array}\right)} .
$$

$$
l+\left(\begin{array}{c}
n+1 \\
2
\end{array}\right)-\left(\begin{array}{c}
l+1 \\
2
\end{array}\right)-N-1-l+2-l+2+N-\left(\begin{array}{l}
n \\
2
\end{array}\right)+\left(\begin{array}{l}
l \\
2
\end{array}\right)=n-2 l+3 .
$$

The right hand side of (4.5) is increased by 2 , so the change of the difference of the sides is $2 l-n-1$, which is non-negative by the supposition $\left\{\frac{n+1}{2}\right\} \leqq l$. Similarly if we are in the interval (4.7), the last terms in (4.5) are

and

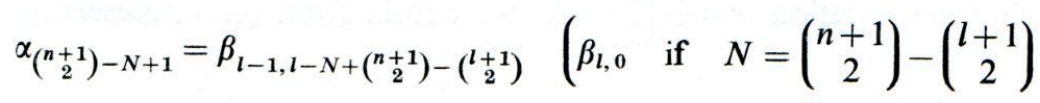

$$
\alpha_{\left(\begin{array}{l}
n \\
2
\end{array}\right)-N+1}=\beta_{l-2, l-1-N+\left(\begin{array}{l}
n \\
2
\end{array}\right)-\left(\begin{array}{l}
l \\
2
\end{array}\right)} .
$$

Thus going to $N+1$, the new terms are

$$
\beta_{l-1, l-N+\left(\begin{array}{c}
n+1 \\
2
\end{array}\right)-\left(\begin{array}{c}
+1 \\
2
\end{array}\right)+1} \text { and } \beta_{l-2, l-N+\left(\begin{array}{c}
n \\
2
\end{array}\right)-\left(\begin{array}{l}
l \\
2
\end{array}\right)} .
$$


Their difference is

$$
l-1+l-N+\left(\begin{array}{c}
n+1 \\
2
\end{array}\right)-\left(\begin{array}{c}
l+1 \\
2
\end{array}\right)+1-l+2-l+N-\left(\begin{array}{l}
n \\
2
\end{array}\right)+\left(\begin{array}{l}
l \\
2
\end{array}\right)=n-l+2 .
$$

The change of the difference of the sides of (4.5) is $l-n$, non-positive because of $l \leqq n$. This proves the statement of our lemma, if

but we need it for $N \leqq \frac{1}{2}\left(\begin{array}{l}n \\ 2\end{array}\right)$. However

$$
N \leqq\left(\begin{array}{l}
n \\
2
\end{array}\right)-\left(\left\{\frac{n+1}{2}\right\}\right)
$$

that is

$$
\frac{1}{2}\left(\begin{array}{l}
n \\
2
\end{array}\right) \leqq\left(\begin{array}{l}
n \\
2
\end{array}\right)-\left(\begin{array}{c}
\frac{n+1}{2} \\
2
\end{array}\right) \text { holds, when } 0 \leqq \frac{1}{2}\left(\begin{array}{l}
n \\
2
\end{array}\right)-\left(\begin{array}{c}
\frac{n+2}{2} \\
2
\end{array}\right) \text {, }
$$

$$
0 \leqq 2 n^{2}-2 n-n^{2}-2 n=n^{2}-4 n,
$$

which is true for $n \geqq 4$. The lemma is proved.
In particular we know that if $C(n, N) \leqq S(n, N)$ holds for $N \leqq \frac{1}{2}\left(\begin{array}{l}n \\ 2\end{array}\right)-\frac{n}{2}$, then it is also true for $n+1$. However, in order to prove Theorem 3 by induction on $n$ we have to prove the inequality for $N \leqq \frac{1}{2}\left(\begin{array}{c}n+1 \\ 2\end{array}\right)-\frac{n+1}{2}$. Also in some cases it is true up to $\frac{1}{2}\left(\begin{array}{c}n+1 \\ 2\end{array}\right)$ and we want to consider those cases as well. This is done in 3 more lemmas. The last one, Lemma 8, gives the complete solution. Since

Instead of $C(n, N) \leqq S(n, N)$ we shall use a further modified version of (4.1).

$$
\sum_{k=1}^{\left(\begin{array}{c}
n \\
2
\end{array}\right)} \alpha_{k}=n\left(\begin{array}{c}
n-1 \\
2
\end{array}\right)
$$

the left hand side of (4.1) is equal to

$$
n\left(\begin{array}{c}
n-1 \\
2
\end{array}\right)-\sum_{k=N+1}^{\left(\begin{array}{c}
n \\
2
\end{array}\right)-N} \alpha_{k}
$$

Introducing the notation $r=\frac{1}{2}\left(\begin{array}{l}n \\ 2\end{array}\right)-N$ we obtain from (4.1) necessarily

$$
\sum_{k=\frac{1}{2}\left(\begin{array}{l}
n \\
2
\end{array}\right)-r+1}^{r-\frac{1}{2}\left(\begin{array}{l}
n \\
2
\end{array}\right)} \alpha_{k} \geqq r(2 n-4),
$$

where $r$ is not necessarily an integer, but there are always $2 r$ terms on the left hand side. 
One more form of $(4.8)$ is

$$
\frac{\sum \alpha_{k}}{r} \geqq 2 n-4
$$

Let $e$ und $f$ be defined by

$$
\frac{1}{2}\left(\begin{array}{l}
n \\
2
\end{array}\right)=\left(\begin{array}{l}
e \\
f
\end{array}\right)+f \quad(0 \leqq f<e) .
$$

(It is easy to see that this is unique).

Lemma 6. Suppose $f<\frac{e}{2}($ in (4.10)) if $2 e+2 f \geqq 2 n-1$. Then $C(n, N) \leqq S(n, N)$ holds when $r=\frac{1}{2}\left(\begin{array}{l}n \\ 2\end{array}\right)-N \leqq e+f-1$.

Proof. Case $A: f>\frac{e}{2}+1$. We want to prove (4.8). The sequence of numbers, we are investigating (when $f$ is an integer) is (starting from left to right and from the middle going backward in the next row).

$$
\left\{\begin{array}{l}
|e-2, \ldots, 2 f-5,2 f-4, \ldots, 2 e-4| e-1, \ldots, e+f-2 \\
2 f \underbrace{-2, \ldots, e+1}_{-e+2 f-2}|\underbrace{2 e, \ldots, 2 f}_{2 e-2 f+1}, 2 \underbrace{2 f-1, \ldots, e}_{2 f-e}| \underbrace{2 e-2, \ldots, e+f-1}_{e-f} .
\end{array}\right.
$$

If $f$ is not an integer, the only difference is, that on the right hand side one number, $e+f-\frac{3}{2}$ stands instead of $e+f-2$ and $e+f-1$.

Observe that in one interval in table (4.11) the sum of the numbers standing under each other is constant. The sums are

$$
\underbrace{e+2 f-4, \ldots}_{-e+2 f-2}, 2 \underbrace{e+2 f-4, \ldots}_{2 e-2 f+1}, \underbrace{e+2 f-2, \ldots}_{-e+2 f}, 2 \underbrace{e+2 f-3, \ldots}_{e-f} .
$$

Subcase 1: Prove the statement for $r=\frac{1}{2}\left(\begin{array}{l}n \\ 2\end{array}\right)-N \leqq e-f$. Using (4.8) we have to prove that the sum of the last $2 r$ terms in (4.11) is not smaller than $r(2 n-4)$. The sum of the last $2 r$ terms of (4.11) is exactly the sum of the last $r$ terms in (4.12). Since $r \leqq e-f$, they are constant. It is enough to prove that

$$
2 e+2 f-3 \geqq 2 n-4 \text {. }
$$

Later we need the inequality

$$
2 e+2 f-4 \geqq 2 n-4
$$

as well, thus we make the calculations here together. From the definition (4.10) of $e$ and $f$ we have

$$
n(n-1)=2 e^{2}-2 e+4 f
$$

Suppose, the converse of (4.13) ((4.14)) is true:

$$
n>e+f+\frac{1}{2} \quad(n>e+f) .
$$


Substituting into (4.15) we obtain

$$
\begin{gathered}
2 e^{2}-2 e+4 f>\left(e+f+\frac{1}{2}\right)\left(e+f-\frac{1}{2}\right) \\
\left(2 e^{2}-2 e+4 f>(e+f)(e+f-1)\right) .
\end{gathered}
$$

Reordering it :

$$
e^{2}-2 e-f(2 e+f-4)+\frac{1}{4}>0 \quad\left(e^{2}-e-f(2 e+f-5)>0\right) .
$$

If $e \geqq 2$ then $2 e+f-4$ (if $e \geqq 3$ then $2 e+f-5$ ) is non negative, $f(2 e+f-4)$ $(f(2 e+f-5))$ is an increasing function of $f$, so (4.16) remains true if we use the inequality $f \geqq \frac{e}{2}$ (supposition of our lemma):

$$
\left\{\begin{array}{l}
e^{2}-2 e-\frac{e}{2}\left(2 e+\frac{e}{2}-4\right)+\frac{1}{4}=-\frac{e^{2}}{4}+\frac{1}{4}>0 \\
\left(e^{2}-e-\frac{e}{2}\left(2 e+\frac{e}{2}-5\right)=-\frac{e^{2}}{4}+\frac{3 e}{2}>0\right) .
\end{array}\right.
$$

However, these inequalities do not hold when $e \geqq 2(e \geqq 6)$. If $n \geqq 3$ then $e \geqq 2$, thus (4.13) is proved for $n \geqq 3$. The inequality (4.14) is proved only for $e \geqq 6$, that is $n \geqq 9$. For the values $n \leqq 3<9$ it is easy to check in Table 1 that the supposition $f \geqq \frac{e}{2}$ of the lemma is not satisfied for $n=3,4,6,7$ and for the remaining values $n=5,8,9$ (4.14) holds.

\begin{tabular}{c|c|c|c}
\hline$n$ & $\frac{1}{2}\left(\begin{array}{l}n \\
2\end{array}\right)$ & $e$ & $f$ \\
\hline 3 & 1,5 & 2 & 0,5 \\
4 & 3 & 3 & 0 \\
5 & 5 & 3 & 2 \\
6 & 7,5 & 4 & 1,5 \\
7 & 10,5 & 5 & 0,5 \\
8 & 14 & 5 & 4 \\
9 & 18 & 6 & 3 \\
10 & 22,5 & 7 & 1,5 \\
11 & 27,5 & 7 & 6,5 \\
12 & 33 & 8 & 5 \\
13 & 39 & 9 & 3 \\
14 & 45,5 & 10 & 0,5 \\
15 & 52,5 & 10 & 7,5 \\
16 & 60 & 11 & 5 \\
17 & 68 & 12 & 2 \\
18 & 76,5 & 12 & 10,5 \\
19 & 85,5 & 13 & 7,5 \\
20 & 95 & 14 & 4 \\
21 & 105 & 15 & 0 \\
22 & 115,5 & 15 & 10,5
\end{tabular}

\begin{tabular}{|l|l|l|l}
\hline$n$ & $\frac{1}{2}\left(\begin{array}{c}n \\
2\end{array}\right)$ & $e$ & $f$ \\
\hline 23 & 126,5 & 16 & 6,5 \\
24 & 138 & 17 & 2 \\
25 & 150 & 17 & 14 \\
26 & 162,5 & 18 & 9,5 \\
27 & 175,5 & 19 & 4,5 \\
28 & 189 & 19 & 18 \\
29 & 203 & 20 & 13 \\
30 & 217,5 & 21 & 7,5 \\
31 & 232,5 & 22 & 1,5 \\
32 & 248 & 22 & 17 \\
33 & 264 & 23 & 11 \\
34 & 280,5 & 24 & 4,5 \\
35 & 297,5 & 24 & 21,5 \\
36 & 315 & 25 & 15 \\
37 & 333 & 26 & 8 \\
38 & 351,5 & 27 & 0,5 \\
39 & 370,5 & 27 & 19,5 \\
40 & 390 & 28 & 12 \\
41 & 410 & 29 & 4 \\
42 & 430,5 & 29 & 24,5 \\
\end{tabular}

Table 1 
Subcase 2: Prove the statement for $e-f<r \leqq f$. The second interval in (4.12) contains the value $e+2 f-2$. If $e+2 f-2 \geqq 2 n-4$, we do not have to prove anything. Otherwise the average $\frac{1}{r} \sum \alpha_{k}$ (see (4.9)) decreases when we use larger values of $r$. So, it is sufficient to prove (4.8) for $r=f$. The left hand side of (4.8) is

$$
(-e+2 f)(e+2 f-2)+(e-f)(2 e+2 f-3)=e^{2}+2 f^{2}-e-f .
$$

(4.8) is equivalent to

$$
e^{2}+2 f^{2}-e-f \geqq f(2 n-4)
$$

Using $e^{2}-e=\left(\begin{array}{l}n \\ 2\end{array}\right)-2 f$ we obtain

and this is equivalent to

$$
\left(\begin{array}{l}
n \\
2
\end{array}\right)+2 f^{2}-3 f \geqq f(2 n-4)
$$

$$
2\left(f-\frac{n}{2}\right)\left(f-\frac{n-1}{2}\right) \geqq 0 .
$$

This inequality always holds, because $f$ is a half of an integer, it cannot satisfy $\frac{n-1}{2}<f<\frac{n}{2}$.

Subcase 3: $f<r \leqq 2 e-f+1$. The new term (see (4.12)) in this interval is $2 e+$ $+2 f-4$. We have proved (4.14), thus the average $\frac{1}{r} \sum \alpha_{k}$ is increasing in this in-
terval, consequently (4.9) and (4.8) hold.

Subcase 4: $2 e-f+1<r \leqq e+f-1$. If the new term $e+2 f-4$ is $\geqq 2 n-4$, we are done. In the contrary case the average is decreasing, so it is sufficient to prove it for $r=e+f-1$. The statement means that the average of $\alpha_{k}^{\prime} \mathrm{s}$ for the 4 intervals is $\geqq 2 n-4$ (see (4.9)). We have proved it for 2 intervals, so it is sufficient to prove the same for the two new intervals:

$$
(-e+2 f-2)(e+2 f-4)+(2 e+2 f-4)(2 e-2 f+1) \geqq(e-1)(2 n-4) .
$$

This is equivalent to

$$
3 e^{2}-2 f \geqq 2 n(e-1) .
$$

Since $f<e$, we can prove (4.18) with $3 e$ instead of $2 f$ :

$$
3 e \geqq 2 n \text {. }
$$

We know $n(n-1)=2 e^{2}-2 e+2 f$ and using again $f<e$ we have

$$
n(n-1)<2 e^{2}+2 e .
$$

Suppose (4.19) does not hold: $e<\frac{2 n}{3}$, and substitute into (4.20):

$$
n(n-1)<\frac{8 n^{2}}{9}+\frac{4 n}{3}, \quad \text { or } \quad n-21<0,
$$


which is a contradiction for $n \geqq 21$. For smaller $n$ 's it is easy to check from Table 1 that (4.19) holds for $n=3,4,6,7,9,10,12,13, \ldots, 20$. For $n=5,8$ and 11 (4.19) is not true, but (4.18) holds. Case A is proved.

Case $B$ : If $\frac{e}{2} \leqq f \leqq \frac{e}{2}+1$, then (4.11) and (4.12) have a slightly different form:

$$
\begin{gathered}
\left\{\begin{array}{l}
|e-2, \ldots, 2 e-4| e-1, \ldots, e+f-2 \\
|2 e, \ldots, e+2 f-2, \ldots, 2 f, 2 f-1, \ldots, e| \\
2 e-2, \ldots, e+f-1,
\end{array}\right. \\
2 \underbrace{e+2 f-4, \ldots}_{e-1}, \underbrace{e+2 f-2, \ldots}_{-e+2 f}, \underbrace{2 e+2 f-3, \ldots}_{e-f} .
\end{gathered}
$$

So, in this case we consider only 3 intervals. The first two are exactly like in Case A. The length of the third is different, but we did not use it. The lemma is proved.

Lemma 7. Suppose $f<\frac{e}{2}$ (in (4.10)). If $2 e+2 f \geqq 2 n-1$, then $C(n, N) \leqq$ $\leqq S(n, N)$ holds when

$$
r=\frac{1}{2}\left(\begin{array}{l}
n \\
2
\end{array}\right)-N \leqq 2 e-f+1 .
$$

If $2 e+2 f<2 n-1$, then $C(n, N) \leqq S(n, N)$ holds when

$$
\min \left(\frac{n}{2}, e-f\right) \leqq r \leqq \begin{cases}2 e+f-3 & (f<2) \\ 2 e-f+1 & (f \geqq 2)\end{cases}
$$

and the opposite inequality holds when $0<r=f$. The inequality $C(n, N) \leqq S(n, N)$ changes its direction only once in this interval.

Proof. Case $A: f \geqq 2$. The sequence of numbers, we are investigating now, have the following form:

$$
\text { (4.21) }\left\{\begin{array}{l}
\ldots, e+2 f-7, \ldots, 2 e-6|e-2, \ldots, e+2 f-3, \ldots, 2 e-4| e-1, \ldots, e+f-2 \\
|\underbrace{2 e, \ldots, e+2 f-1}_{e-2 f+2}, \underbrace{e+2 f-2, \ldots, e \mid}_{2 f-1}| \underbrace{2+2, \ldots, e+2 f-1}_{e-2 f}, \underbrace{e+2 f-2, \ldots, e+f-1}_{f} .
\end{array}\right.
$$

Observe that in one interval in table (4.21) the sum of the numbers standing under each other is constant. The sums are

$$
3 \underbrace{e+2 f-7, \ldots}_{e-2 f+2}, \underbrace{2 e+2 f-4, \ldots}_{2 f-1}, \underbrace{3 e+2 f-5}_{e-2 f}, \ldots \underbrace{2 e+2 f-3, \ldots}_{f} .
$$

Subcase 1. If $2 e+2 f-3 \geqq 2 n-4$, then (4.8) holds for $0<r \leqq f$, otherwise it does not hold. This proves the lemma for the first interval.

Subcase 2. Consider now the interval $f<r \leqq e-f$. If $2 e+2 f-3 \geqq 2 n-4$ is true, $3 e+2 f-5 \geqq 2 n-4$ is also true whenever $e \geqq 2$ (that is, $n \geqq 3$ ). Then (4.8) holds. If $2 e+2 f-3<2 n-4$ holds, then first we prove that (4.8) holds for $r=e-f$. We have to prove

$$
(e-2 f)(3 e+2 f-.5)+f(2 e+2 f-3) \geqq(e-f)(2 n-4),
$$


that is,

(4.23)

$$
3 e^{2}-2 f^{2}-2 e f-e+3 f \geqq 2 n(e-f) .
$$

We use $e^{2}=\left(\begin{array}{l}n \\ 2\end{array}\right)+e-2 f$ :

$$
3\left(\begin{array}{l}
n \\
2
\end{array}\right)-2 f^{2}-2 e f+2 e-3 f \geqq 2 n(e-f) .
$$

By the suppositions $2 e+2 f \leqq 2 n-2$, so it is enough to prove this last inequality after substituting $-f(2 e+2 f)$ by $-f(2 n-2)$ :

$$
3\left(\begin{array}{l}
n \\
2
\end{array}\right)-2 n f+2 f+2 e-3 f \geqq 2 n(e-f)
$$

or

Since $f<e$, we can write

$$
3\left(\begin{array}{l}
n \\
2
\end{array}\right)+2 e-f \geqq 2 n e .
$$

$$
3\left(\begin{array}{l}
n \\
2
\end{array}\right)+e \geqq 2 n e .
$$

We prove it in an indirect way. Suppose the contrary, i.e.

$$
e>\frac{3\left(\begin{array}{l}
n \\
2
\end{array}\right)}{2 n-1}
$$

and use $\left(\begin{array}{l}n \\ 2\end{array}\right)=e^{2}-e+2 f \geqq e^{2}-e$. As the right hand side of this inequality is increasing in $e$, it follows

$$
\left(\begin{array}{l}
n \\
2
\end{array}\right)>\frac{3\left(\begin{array}{l}
n \\
2
\end{array}\right)}{2 n-1}\left(\frac{3\left(\begin{array}{l}
n \\
2
\end{array}\right)}{2 n-1}-1\right)
$$

by (4.25). Reordering it, we obtain $0>n^{2}-13 n+4$. This is a contradiction for $n \geqq 13$. For smaller $n$ 's: (4.26) holds (see Table 1) when $n=5,6,8,9,10,11,12$. For $n=3,4,7(4.24)$ is not true, but (4.23) is true.

Subcase $2 a$. If $\frac{n}{2}<e-f$, we have to prove that the inequality (4.8) changes its direction earlier than $e-f$, at $\frac{n}{2}$. It is enough to prove that it holds for $r=\frac{n}{2}$.
In other words:

$$
\left(\frac{n}{2}-f\right)(3 e+2 f-5)+f(2 e+2 f-3) \geqq(2 n-4) \frac{n}{2}
$$

has to be proved, or equivalently

$$
3 n e+2 n f-2 e f+4 f \geqq 2 n^{2}+n .
$$

It is sufficient to prove $3 n e \geqq 2 n^{2}+n$ (we omitted a positive number, as $n \geqq e$ ), or

$$
3 e \geqq 2 n+1 \text {. }
$$


We verify it in an indirect way. Suppose $e<\frac{2 n+1}{3}$, and substitute into the inequality

$$
\begin{gathered}
n^{2}-n=2 e^{2}-2 e+4 f<2 e^{2}+2 e=2 e(e+1), \\
n^{2}-n<2 \frac{2 n+1}{3} \cdot \frac{2 n+4}{3},
\end{gathered}
$$

or equivalently $n^{2}-29 n-8<0$ which is a contradiction if $n \geqq 30$. For smaller $n$ 's: (4.27) holds (see Table 1) when $n=4,7,10,13,14,16,17,19,20,21,22$, $23,24,25,26,27,28,29$. The remaining $n$ 's do not belong to this case $\left(f<\frac{e}{2}, \frac{n}{2}<e-f\right)$.

Subcase 3: $e-f<r \leqq e+f-1$. If $2 e+2 f-4 \geqq 2 n-4$, the averages do not decrease in this interval, and we are done. If $2 e+2 f-4<2 n-4$, it is enough to investigate $r=e+f-1$, the average will be the smallest here. We have to prove that the sum in the last 3 small intervals is $\geqq(e+f-1)(2 n-4)$. The $3^{d}$ interval gives

$$
(2 f-1)(2 e+2 f-4)=4 e f+4 f^{2}-2 e-10 f+4 \text {. }
$$

Adding to the sum of the 2 previous ones (see (4.23)):

or equivalently

$$
3 e^{2}+2 f^{2}+2 e f-7 e-3 f+4 \geqq(e+f-1)(2 n-4)
$$

$$
3 e^{2}-3 e \geqq 2 e n-2 n+f(2 n-2 f-2 e-1) .
$$

It is easy to see, that

$$
\begin{aligned}
f(2 n-2 f-2 e-1) & =\frac{1}{2} 2 f(2 n-2 f-2 e-1) \leqq \frac{1}{2}\left(\frac{2 n-2 e-1}{2}\right)^{2}= \\
& =\frac{n^{2}}{2}+\frac{e^{2}}{2}+\frac{1}{8}-n e-\frac{n}{2}+\frac{e}{2} .
\end{aligned}
$$

Substitute it into (4.28)

or equivalently

$$
6 e^{2}-6 e \geqq 4 e n-4 n+n^{2}+e^{2}+\frac{1}{4}-2 n e-n+e
$$

$$
-n^{2}+5 e^{2}-2 e n-7 e+5 n-\frac{1}{4} \geqq 0
$$

Use $e^{2}=\left(\begin{array}{l}n \\ 2\end{array}\right)+e-2 f$ :

$$
3 n^{2}-4 e n-4 e+5 n-20 f-\frac{1}{2} \geqq 0 .
$$

Since $2 f<e$ :

$$
3 n^{2}-4 e n-14 e+5 n-\frac{1}{2} \geqq 0
$$


From $\left(\begin{array}{l}n \\ 2\end{array}\right)=e^{2}-e+2 f$ we obtain

Substitute it into

$$
e=\frac{1+\sqrt{1-8 f+4\left(\begin{array}{l}
n \\
2
\end{array}\right)}}{2}<\frac{1+\sqrt{2 n^{2}}}{2}=\frac{1+n \sqrt{2}}{2}
$$

$$
(3-2 \sqrt{2}) n^{2}-(7 \sqrt{2}-3) n-7-\frac{1}{2} \geqq 0 .
$$

This holds for $n \geqq 42$. An ugly compution shows, that for $3 \leqq n \leqq 41, n \neq 6$, either (4.29) holds or $f \geqq \frac{e}{2}$, that is, not our case. For $n=6$ (4.28) holds.

Subcase 4: $e+f-1<r \leqq 2 e-f+1$. If

$$
3 e+2 f-7 \geqq 2 n-4,
$$
then the new terms do not decrease the average $\frac{1}{r} \sum \alpha_{k}$. Use the assumption $f \geqq 2$
of Case A:

$$
3 e+1 \geqq 2 n .
$$

This is weaker than (4.29), which was proved for $n \geqq 21$ and was checked for $n=3,4,6,7,9,10,12,13, \ldots, 20$. For $n=5,8$, and 11 (4.32) holds.

Case $B: 0<f<2$. (4.21) and (4.22) have a slightly modified form

$$
\begin{gathered}
\left\{\begin{array}{c}
\ldots|e-3, \ldots, 2 e-6| e-2, \ldots, e+2 f-4, e+2 f-3-4 \mid e-1, \ldots, e+f-2 \\
|\underbrace{2 e, \ldots, e+2 f-1}_{e-2}, e+\underbrace{2 f-2, \ldots,}_{2 f-1} e| \underbrace{2 e-2, \ldots, e+2 f-1,}_{e-2 f} \underbrace{e+2 f-2, \ldots, e+f-1}_{f}
\end{array}\right. \\
\underbrace{3 e+2 f-7, \ldots}_{e-2}, 2 \underbrace{2 e+2 f-4, \ldots}_{2 f-1}, 3 \underbrace{3 e+2 f-5, \ldots}_{e-2 f}, \underbrace{2 e+2 f-3, \ldots}_{f} .
\end{gathered}
$$

The only change that the $4^{\text {th }}$ interval is shorter, but we did not use its length. The cases $n=3$ and 7 can be done by an easy computation.

Case $C: f=0$.

$$
\begin{aligned}
& \left\{\begin{array}{l}
\ldots, 2 e-8|e-3, \ldots, 2 e-7,2 e-6| e-2, \ldots, 2 e-4 \mid \\
|2 e, 2 e-1,2 e-2,2 e-3, \underbrace{2 e-4, \ldots}_{e-3}, e| \underbrace{2 e-2}_{1}, 2 \underbrace{2 e-3, \ldots, e-1}_{e-1}
\end{array}\right. \\
& \underbrace{3 e-7, \ldots}_{e-3}, \underbrace{4 e-8, \ldots}_{1}, \underbrace{3 e-5, \ldots}_{e-1} .
\end{aligned}
$$

We prove that all these numbers are $\geqq 2 n-4$. It is enough to prove that $3 e-7 \geqq$ $\geqq 2 n-4$, that is $3 e-3 \geqq 2 n$. We prove it in an indirect way. Suppose $e<\frac{2 n+3}{3}$ 
and use $n(n-1)=2 e(e-1)$ :

$$
n(n-1)<2 \frac{2 n+3}{3} \cdot \frac{2 n}{3}
$$

or equivalently $n-21<0$. This is a contradiction if $n \geqq 21$. There is only one case, when $n<21$ and $f=0$ (see Table 1), namely when $n=4$. It is easy to check, that the statement holds for $n=4$.

LEMMA 8. If $f \geqq \frac{e}{2}$ in (4.10), then

$$
C(n, N) \leqq S(n, N) \quad \text { for } \quad 0 \leqq N \leqq \frac{1}{2}\left(\begin{array}{l}
n \\
2
\end{array}\right)
$$

and

$$
C(n, N) \geqq S(n, N) \quad \text { for } \quad \frac{1}{2}\left(\begin{array}{l}
n \\
2
\end{array}\right) \leqq N \leqq\left(\begin{array}{l}
n \\
2
\end{array}\right)
$$

If $f<\frac{e}{2}$, but $2 e+2 f \geqq 2 n-1$, then (4.33) and (4.34) hold, again. If $f<\frac{e}{2}, 2 e+2 f<$ $<2 n-1$ then there is an $R$ such that $f \leqq R \leqq \min \left(\frac{n}{2}, e-f\right)$, and

$$
\begin{gathered}
C(n, N) \leqq S(n, N) \text { for } 0 \leqq N \leqq \frac{1}{2}\left(\begin{array}{l}
n \\
2
\end{array}\right)-R \\
C(n, N) \geqq S(n, N) \text { for } \quad \frac{1}{2}\left(\begin{array}{l}
n \\
2
\end{array}\right)-R \leqq N \leqq \frac{1}{2}\left(\begin{array}{l}
n \\
2
\end{array}\right) \\
C(n, N) \leqq S(n, N) \text { for } \frac{1}{2}\left(\begin{array}{l}
n \\
2
\end{array}\right) \leqq N \leqq \frac{1}{2}\left(\begin{array}{l}
n \\
2
\end{array}\right)+R \\
C(n, N) \geqq S(n, N) \text { for } \frac{1}{2}\left(\begin{array}{l}
n \\
2
\end{array}\right)+R \leqq N \leqq\left(\begin{array}{l}
n \\
2
\end{array}\right) .
\end{gathered}
$$

Proof. We use induction over $n$. For $n=3$ the statements are true. Let $n>3$. Suppose the statement is true for $n-1$, we want to prove it for $n$. According to the induction hypothesis $C(n-1, N) \leqq S(n, N)$ if

$$
0 \leqq N \leqq \frac{1}{2}\left(\begin{array}{c}
n-1 \\
2
\end{array}\right)-\frac{n-1}{2} .
$$

By Lemma 5

$$
C(n, N) \leqq S(n, N)
$$

follows under the condition (4.35). From Lemma 6 we know that (4.36) holds when

$$
\frac{1}{2}\left(\begin{array}{l}
n \\
2
\end{array}\right)-z \leqq N \leqq \frac{1}{2}\left(\begin{array}{l}
n \\
2
\end{array}\right)-R,
$$


where

$$
z=\left\{\begin{array}{lll}
e+f-1 & \text { if } & \frac{e}{2} \leqq f \\
2 e-f+1 & \text { if } & 2 \leqq f<\frac{e}{2} \\
2 e+f-3 & \text { if } & 0 \leqq f<2
\end{array}\right.
$$

and $R=0$ if $\frac{e}{2} \leqq f$,

$$
f \leqq R \leqq \min \left(\frac{n}{2}, e-f\right) \text { if } \frac{e}{2}>f .
$$

If we are able to prove that

$$
\frac{1}{2}\left(\begin{array}{l}
n \\
2
\end{array}\right)-z \leqq \frac{1}{2}\left(\begin{array}{c}
n-1 \\
2
\end{array}\right)-\frac{n-1}{2}
$$

(see (4.35) and (4.37)), then (4.36) holds under the condition

(4.39) is equivalent to

$$
0 \leqq N \leqq \frac{1}{2}\left(\begin{array}{l}
n \\
2
\end{array}\right)-R .
$$

$$
n-1 \leqq z .
$$

Case $A: \frac{e}{2} \leqq f$. Then $z=e+f-1$. Assume the opposite of (4.40) holds: $n-1>e+f-1$ or $n>e+f$. Substitute it into the equality $n(n-1)=2 e^{2}-2 e+4 f$ :

which is equivalent to

$$
(e+f)(e+f-1)<2 e^{2}-2 e+4 f,
$$

$$
e^{2}-f^{2}-2 e f-e+5 f>0 .
$$

If $f+2 e-5 \geqq 0$ (if $e \geqq 2$ ), then

$$
f(f+2 e-5) \geqq \frac{e}{2}(f+2 e-5) \geqq \frac{e}{2}\left(\frac{e}{2}+2 e-5\right)=\frac{5 e^{2}}{4}-\frac{5 e}{2},
$$

(4.41) results in $-e^{2}+6 e>0$. This is a contradiction if $e \geqq 6$, that is, if $n \geqq 9$. For smaller $n$ 's either $\frac{e}{2}>f$ or (4.40) holds.

Case $B: 2 \leqq f<\frac{e}{2}$. We have to prove $n-1 \leqq 2 e-f+1$ or $2 n \leqq 3 e+4$. Use

$$
\begin{gathered}
\frac{n-1}{2}<\sqrt{\left(\begin{array}{l}
n \\
2
\end{array}\right)}=\sqrt{e^{2}-e+2 f} \leqq e: \\
2 n \leqq \frac{3 n}{\sqrt{2}}-\frac{3}{\sqrt{2}}+4,
\end{gathered}
$$

which holds for $n \geqq 0$. 
Case $C: 0 \leqq f<2$. We have to prove $n-1 \leqq 2 e+f-3$ or

$$
n \leqq 2 e-2 \text {. }
$$

Use $\frac{n-1}{\sqrt{2}} \leqq e: n \leqq \frac{2 n-2}{\sqrt{2}}-2$. This holds for $n \geqq 9$. For $n=4,6$ and 7 ( $n=5$ and 8 do not belong to this case) (4.42) holds. The lemma is proved. Lemma 8 completely proves our Theorem 3 .

\section{The second proof of Theorem 2}

We use the results of Section 3, in particular Lemma 8, and its methods and notation. First we have to prove another inequality:

LEMMA 9. $C(n, N+n)-C(n, N) \geqq 2 N+\left(\begin{array}{l}n \\ 2\end{array}\right)$ if

$$
\left(\left[\begin{array}{c}
\frac{n}{2} \\
2
\end{array}\right] \leqq N \leqq\left(\begin{array}{l}
n \\
2
\end{array}\right) .\right.
$$

Proof. We start with the case $N=\left(\begin{array}{l}l \\ 2\end{array}\right)\left(\left[\frac{n}{2}\right] \leqq 1 \leqq n\right)$. Then the statement holds with equality. The difference $C(n, N+n)-C(n, N)$ is the sum of the following terms (see Lemma 4):

$$
\beta_{l-1,0}, \ldots, \beta_{l-1, l-1}, \beta_{l, 0}, \ldots, \beta_{l, n-l-1}
$$

(here we used $1 \leqq n$ and $n \leqq 2 l+1$ ). The sum of these terms is

$$
\sum_{i=l-1}^{2 l-2} i+\sum_{i=l}^{n-1} i=3\left(\begin{array}{l}
l \\
2
\end{array}\right)+(n-l) \frac{n+l-1}{2}=2\left(\begin{array}{l}
l \\
2
\end{array}\right)+\left(\begin{array}{l}
n \\
2
\end{array}\right) .
$$

The desired equality is proved. Now we prove that the function

$$
C(n, N+n)-C(n, N)-2 N-\left(\begin{array}{l}
n \\
2
\end{array}\right)
$$

is increasing in the interval

and it is decreasing in

$$
\left(\begin{array}{l}
l \\
2
\end{array}\right) \leqq N \leqq\left(\begin{array}{l}
l \\
2
\end{array}\right)+2 l+1-n
$$

$$
\left(\begin{array}{l}
l \\
2
\end{array}\right)+2 l+1-n \leqq N \leqq\left(\begin{array}{c}
l+1 \\
2
\end{array}\right) \quad\left(\left[\frac{n}{2}\right\rceil \leqq l<n\right) .
$$

This proves the statement, since (5.1) is 0 for $N=\left(\begin{array}{l}l \\ 2\end{array}\right)$ and $N=\left(\begin{array}{c}l+1 \\ 2\end{array}\right)$. If

$$
\left(\begin{array}{l}
l \\
2
\end{array}\right) \leqq m<\left(\begin{array}{l}
l \\
2
\end{array}\right)+2 l+1-n,
$$


then the difference $C(n, N+n)-C(n, N)$ is the sum of the terms

Here

$$
\beta_{l-1, N-\left(\begin{array}{l}
l \\
2
\end{array}\right)}, \ldots, \beta_{l-1, l-1}, \beta_{l, 0}, \ldots, \beta_{l, n-l-1+N-\left(\begin{array}{l}
l \\
2
\end{array}\right)} .
$$

and

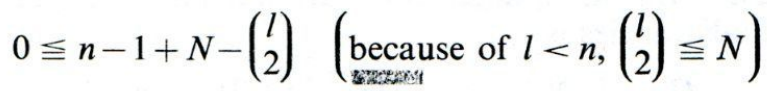

$$
n-l-1+N-\left(\begin{array}{l}
l \\
2
\end{array}\right)<l .
$$

If we change $N$ to $N+1$, a new term comes in: $\beta_{l, n-l+N\left(\frac{(}{2}\right)}$, and $\beta_{l-1, N-\left(\begin{array}{l}l \\ 2\end{array}\right)}$ wil be omitted. The change is

$$
n+N-\left(\begin{array}{l}
l \\
2
\end{array}\right)-l+1-N+\left(\begin{array}{l}
l \\
2
\end{array}\right)=n-l+1 .
$$

However, in $(5.1) 2 N$ changes to $2(N+1)$. So, the total change is $n-l-1 \geqq 0$, and the function (5.1) is increasing. On the other hand, if

$$
\left(\begin{array}{l}
l \\
2
\end{array}\right)+2 l+1-n \leqq N<\left(\begin{array}{c}
l+1 \\
2
\end{array}\right),
$$

then the difference $C(n, N+n)-C(n, N)$ is the sum of the terms

where

$$
\beta_{l-1, N-\left(\begin{array}{l}
l \\
2
\end{array}\right)}, \ldots, \beta_{l-1, l-1}, \beta_{l, 0}, \ldots, \beta_{l, l}, \beta_{l+1,0}, \ldots, \beta_{l+1, n+2 l+N-\left(\begin{array}{l}
l \\
2
\end{array}\right)},
$$

$$
-1 \leqq n-2 l-2+N-\left(\begin{array}{l}
l \\
2
\end{array}\right)<l+1
$$

(using $N<\left(\frac{l+1}{2}\right)$ and $n \leqq 2 l+1$, if $=-1, b_{l, l}$ is the last term). Changing $N$ into $N+1$, the sum changes with

$$
\beta_{l+1, n-2 l-1+N-\left(\begin{array}{l}
l \\
2
\end{array}\right)}-\beta_{l-1, N-\left(\begin{array}{l}
l \\
2
\end{array}\right)}=n-2 l+1,
$$

and (5.1) changes with $n-2 l-1 \leqq 0$. The function is decreasing in this interval. The lemma is proved.

LEMMA 10.

$$
f(n, N)=\max \left(f(n-1, N), f(n-1, N-n+1)+\left(\begin{array}{c}
n-1 \\
2
\end{array}\right)+2(N-n+1)\right)
$$

if $n-1 \leqq N \leqq\left(\begin{array}{c}n-1 \\ 2\end{array}\right)$. (Otherwise only the defined term is considered on the right hand side.)

Proof. Consider an optimal graph. There are two possibilities: a) either each vertex is contained in at least one edge b) or not.

In case b) our graph must be optimal also for $n-1$ vertices, so $f(n, N)=$ $=f(n-1, N)$. In Case a) there is an edge containing the last vertex, thus by Lemma 1 , the first vertex is connected with every vertex. Consequently, at the 
first point there are $\left(\begin{array}{c}n-1 \\ 2\end{array}\right)$ adjacencies. The remaining $N-n+1$ edges have one adjacency at their both ends with edges going to the first vertex. This is $2(N-n+1)$. What remained is the number of adjacencies among edges not containing the first vertex. They must form an optimal configuration on $n-1$ points. Thus in case a) the number of adjacencies is

The lemma is proved.

$$
f(n-1, N-n+1)+\left(\begin{array}{c}
n-1 \\
2
\end{array}\right)+2(N-n+1) .
$$

Proof of Theorem 3. We prove that $f(n, N)=C(n, N)$ or $S(n, N)$ by induction on $n$. The exact result is given by Lemma 8 . This is true for $n=2,3$. Suppose it is also true for $n-1$ (with any $N$ ) and let us prove it for $n$.

Case $A$. Suppose $f(n-1, N)$ and $f(n-1, N-n+1)$ are both assumed for quasi-complete graphs. Since $N \leqq\left(\begin{array}{l}n \\ 2\end{array}\right)$, also $N-n+1 \leqq\left(\begin{array}{c}n-1 \\ 2\end{array}\right)$.

Subcase 1. If additionally

$$
\left(\left[\begin{array}{c}
\frac{n-1}{2} \\
2
\end{array}\right] \leqq N-n+1 \leqq\left(\begin{array}{c}
n-1 \\
2
\end{array}\right),\right.
$$

then we can use Lemma 9:

$$
C(n-1, N)-C(n-1, N-n+1) \geqq\left(\begin{array}{c}
n-1 \\
2
\end{array}\right)+2(N-n+1) .
$$

That is the first term under the max in (5.2) is $\geqq$ than the other one. Consequently $f(n, N)$ is assumed for the quasi-complete graph.

Subcase 2: $N-n+1<\left(\left[\begin{array}{c}\frac{n-1}{2} \\ 2\end{array}\right)\right.$. In this case we shall prove that $C(n-1$, $N-n+1) \leqq S(n-1, N-n+1)$. Indeed, it follows from Lemma 8 , when

$$
\left(\left[\begin{array}{c}
\frac{n-1}{2} \\
2
\end{array}\right]\right)-1 \leqq \frac{1}{2}\left(\begin{array}{c}
n-1 \\
2
\end{array}\right)-\frac{n-1}{2},
$$

that is always for $n \geqq 4$. It means that in Subcase $2, f(n-1, N-n+1)$ is assumed by the quasi-star and it belongs to the next case.

Case B: Suppose $f(n-1, N)$ is assumed for the quasi-complete graph and $f(n-1, N-n+1)$ for the quasi-star. It is easy to see that

$$
S(n-1, N-n+1)+\left(\begin{array}{c}
n-1 \\
2
\end{array}\right)+2(N-n+1)=S(n, N) .
$$

In (5.2) we have $\max (C(n-1, N), S(n, m))$, we obtain always either a quasi-star or a quasi-complete graph. 
Case $C: f(n-1, N)$ is assumed strictly for the quasi-star and $f(n-1, N-n+1)$ strictly for the quasi-complete graph. It means that

$$
C(n-1, N)<S(n-1, N)
$$

and

$$
C(n-1, N-n+1)>S(n-1, N-n+1) .
$$

By Lemma 8 (5.3) results in

and (5.4) results in

$$
N<\frac{1}{2}\left(\begin{array}{c}
n-1 \\
2
\end{array}\right)+\frac{n-1}{2}
$$

$$
\frac{1}{2}\left(\begin{array}{c}
n-1 \\
2
\end{array}\right)-\frac{n-1}{2}<N-n+1 .
$$

But (5.5) and (5.6) contradict each other.

Case D: $f(n-1, N)$ is assumed strictly for the quasi-star and $f(n-1, N-n+1)$ (not necessarily strictly) also for the quasi-star. We have again (5.3) and (5.5). We shall prove that in (5.2) the second term under the maximum is larger, that is, $S(n-1, N) \leqq S(n, N)$. Rewrite this inequality using Lemma 3:

or

$$
\begin{gathered}
C\left(n-1,\left(\begin{array}{c}
n-1 \\
2
\end{array}\right)-N\right)-(n-1)\left(\begin{array}{c}
n-2 \\
2
\end{array}\right)-4 N+2(n-1) N \leqq \\
\leqq C\left(n,\left(\begin{array}{l}
n \\
2
\end{array}\right)-N\right)-n\left(\begin{array}{c}
n-1 \\
2
\end{array}\right)-4 N+2 n N,
\end{gathered}
$$

$$
\begin{gathered}
C\left(n-1,\left(\begin{array}{l}
n \\
2
\end{array}\right)-N\right)-C\left(n-1,\left(\begin{array}{c}
n-1 \\
2
\end{array}\right)-N\right) \geqq n\left(\begin{array}{c}
n-1 \\
2
\end{array}\right)-(n-1)\left(\begin{array}{c}
n-2 \\
2
\end{array}\right)-2 N= \\
=\left(\begin{array}{c}
n-1 \\
2
\end{array}\right)+2\left(\left(\begin{array}{c}
n-1 \\
2
\end{array}\right)-N\right) .
\end{gathered}
$$

By Lemma 10 this holds when

$$
\left(\left[\begin{array}{c}
\frac{n-1}{2} \\
2
\end{array}\right] \leqq\left(\begin{array}{c}
n-1 \\
2
\end{array}\right)-m \leqq\left(\begin{array}{c}
n-1 \\
2
\end{array}\right)\right.
$$

or

$$
0 \leqq m \leqq\left(\begin{array}{c}
n-1 \\
2
\end{array}\right)-\left(\left[\begin{array}{c}
\frac{n-1}{2} \\
2
\end{array}\right] .\right.
$$

However, this follows from (1.2) when

$$
\frac{1}{2}\left(\begin{array}{c}
n-1 \\
2
\end{array}\right)+\frac{n-1}{2} \leqq\left(\begin{array}{c}
n-1 \\
2
\end{array}\right)-\left(\left[\begin{array}{c}
\frac{n-1}{2} \\
2
\end{array}\right]\right)+1
$$

holds, that is always if $n \geqq 4$. The theorem is finally proved. 


\section{Open questions}

1. Some strange number-theoretical combinatorial questions arise. What is the relative density of the numbers $n$ for which $R=0$ (see Lemma 8)? What is the distribution of $\frac{R}{n}$ ?

2. We started to think about the next problem. $N\left(0 \leqq N \leqq\left(\begin{array}{l}n \\ 3\end{array}\right)\right)$ different 3-types are given on an $n$-set. What is the maximal number of pairs of 3-tuples having 2 elements in common. By easy symmetry-arguments it is enough to consider the case $0 \leqq N \leqq \frac{1}{2}\left(\begin{array}{l}n \\ 3\end{array}\right)$. We conjecture that in this interval the maximum is assumed for a quasi-complete 3-graph, or a quasi-3-star on some $m \leqq n$ elements. Recently Vera T. Sós and M. Simonovits have some results in connection with this problem.

\section{Reference}

[1] Moshe Katz, Rearrangements of (0-1) matrices, Israel J. Math., 9 (1971). 53-72.

(Received January 17, 1977)

MATHEMATICAL INSTITUTE

OF THE HUNGARIAN ACADEMY OF SCIENCES

1053 BUDAPEST, REÁLTANODA U. 13-15. 